\section{$c 6$ \\ the DNA \\ methylation \\ landscape, \\ although \\ mainly \\ static, does \\ vary across \\ generations

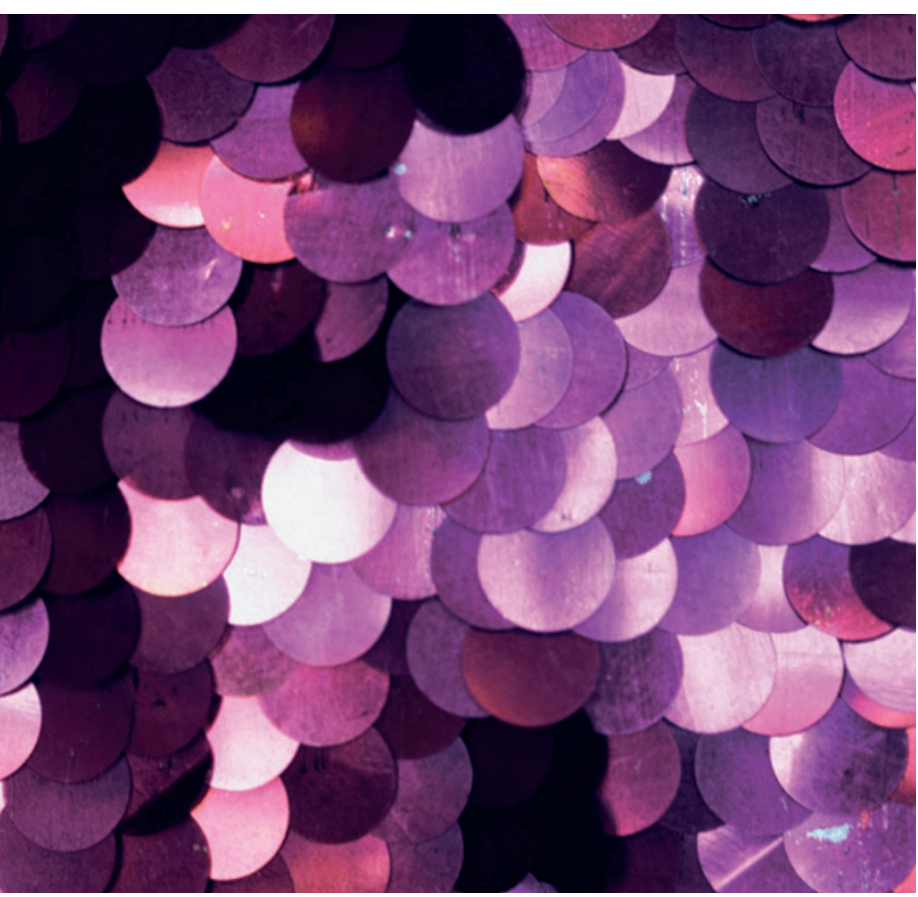

EPIGENOMICS

\title{
Epigenetic variation across the generations
}

Advances in DNA-sequencing technologies have driven the cataloguing of genetic variation in humans and an increasing number of model organisms. The same technologies are now being extended to epigenetic modifications. Two reports - from Becker and Hagmann et al. in Nature and Schmitz et al. in Science - provide the first genome-wide insights into heritable epigenetic variation. Studying DNA methylation in Arabidopsis thaliana, both groups show that transgenerational epigenetic variation is likely to have an important role in generating sequence-independent epialleles that can be transmitted from parents to offspring.

A handful of local, inherited DNA methylation variants that affect the transcription of nearby genes have been described in plants before. However, the rate of emergence of these epialleles, and their stability, is unclear. To address these issues, the two groups turned to mutation accumulation lines in A. thaliana that had been grown for 30 generations in a common environment. Singlebase-pair resolution methylomes were generated for the parental and descendant generations, and comparisons were made both at single-nucleotide positions and at differentially methylated regions (DMRs), which are larger regions of contiguous DNA methylation.

The overall picture was clear: the DNA methylation landscape, although mainly static, does vary across generations. Schmitz et al. estimate the 'epimutation rate' to be $4 \times 10^{-4}$ per CG site per generation. The spontaneous genetic mutation rate had been calculated at $7 \times 10^{-9}$ base substitutions per site per generation in the same A. thaliana lines. Becker and Hagmann et al. also compared parents and their immediate progeny. Surprisingly, the epimutation rate between each generation is $\sim 3-4$ times higher than the longer-term rate. These authors propose that this difference is due to frequent reversions and that epialleles are more likely to contribute to short-term inheritance than long-term evolution.

Interesting insights came from looking at the genome-wide distribution of this epigenetic variation. Becker and Hagmann et al. point out that CG methylation of transposable-element-rich regions appears to be more stable than that of protein-coding genes. Although the mechanisms underlying the generation of epigenetic variation remain to be elucidated, these authors point out that this genomic distribution might hold the first clue. As variable methylation sites tend to lie away from transposable elements, which are also the sites to which small interfering RNAs (siRNAs) map, it may well be that siRNAs have a role in stabilizing methylation. Taking a developmental perspective, Schmitz et al. propose that epimutations might arise during epigenetic reprogramming that occurs after fertilization.

Both groups also saw changes between generations in DNA methylation at DMRs. Becker and Hagmann et al. discovered that even such large-scale changes can occur within a single generation. That said, their estimates suggest that these larger regions change at a slower rate, which is more similar to the genetic mutation rate.

The phenotypic impact of these epimutations is illustrated by mRNA expression analyses. Both groups report expression changes across the generations at several of the loci affected by DNA methylation changes. In addition to these molecular differences, it has been reported before that there are subtle morphological differences between these mutation accumulation lines. This raises the possibility that individual methylation variants could be linked to specific traits using quantitative genetics approaches. The association of epimutations to specific phenotypes, as well as the molecular mechanisms that generate and maintain them, will undoubtedly be the two most hotly pursued goals for the near future.

Magdalena Skipper, Senior Editor, Nature

ORIGINAL RESEARCH PAPERS Becker, C. et al. Spontaneous epigenetic variation in the Arabidopsis thaliana methylome. Nature $20 \mathrm{Sep}$ 2011 (doi:10.1038/nature10555)|Schmitz, R. J. et al. Transgenerational epigenetic instability is a source of novel methylation variants. Science 15 Sep 2011 (doi:10.1126/science.1212959) 